\title{
Preserved efficacy and reduced toxicity with intermittent linezolid dosing in combination with bedaquiline and pretomanid in a murine TB model
}

Kristina M. Bigelow ${ }^{1}$, Rokeya Tasneen ${ }^{2}$, Yong S. Chang ${ }^{2}$, Kelly E. Dooley ${ }^{1,2}$ and Eric L. Nuermberger ${ }^{2}$

${ }^{1}$ Department of Pharmacology and Molecular Sciences, Johns Hopkins University School of Medicine,

Baltimore 21287, USA

${ }^{2}$ Center for Tuberculosis Research, Department of Medicine, Johns Hopkins University School of

Medicine, Baltimore 21287, USA

Running Head (limit 54 characters/spaces): Linezolid PK-PD and PK-TD in preclinical TB model

Corresponding Author: Eric L. Nuermberger

(410) 502-0580 / FAX (410) 614-8173

E-mail: enuermb@jhmi.edu

Key Words (Limit 3-10): Pretomanid, bedaquiline, linezolid, toxicity, pharmacokinetic, animal model 


\begin{abstract}
The novel regimen of bedaquiline, pretomanid, and linezolid (BPaL) is highly effective against drug resistant tuberculosis, but linezolid toxicities are frequent. We hypothesized that, for a similar total weekly cumulative dose, thrice-weekly administration of linezolid would preserve efficacy while reducing toxicity compared to daily dosing, in the context of the BPaL regimen.
\end{abstract}

Using $\mathrm{C} 3 \mathrm{HeB} / \mathrm{FeJ}$ and $\mathrm{BALB} / \mathrm{c}$ mouse models of tuberculosis disease, thrice-weekly linezolid dosing was compared to daily dosing, with intermittent dosing introduced (a) from treatment initiation or (b) following an initial period of daily dosing. In all animals, BPa was dosed daily throughout treatment. Blood counts were used to assess hematologic toxicity. Following unexpected findings of apparent antagonism, we conducted additional experiments to investigate strain-to-strain differences in the contribution of linezolid to regimen efficacy comparing each 1- and 2-drug component to the BPaL regimen in BALB/c mice infected with Mycobacterium tuberculosis H37Rv or HN878.

Giving linezolid daily for 1-2 months achieved the greatest efficacy, but following that, results were similar if the drug was stopped, dosed thrice-weekly, or continued daily. Erythrocyte counts were lower with daily than thrice-weekly dosing. Linezolid had additive effects with BPa against M. tuberculosis H37Rv but antagonistic effects with BPa against M. tuberculosis HN878. However, overall efficacy of BPaL was high and similar against both strains. 
bioRxiv preprint doi: https://doi.org/10.1101/2020.06.10.145334; this version posted June 12, 2020. The copyright holder for this preprint (which was not certified by peer review) is the author/funder, who has granted bioRxiv a license to display the preprint in perpetuity. It is made available under aCC-BY-NC-ND 4.0 International license.

Dosing linezolid daily for the first two months, then less frequently thereafter, may optimize its therapeutic margin. Linezolid's contribution to BPaL regimens may depend on M. tuberculosis strain. 


\section{INTRODUCTION}

Tuberculosis (TB) is the leading cause of death by a single infectious agent worldwide.

3

4
While treatment of drug-susceptible TB is relatively effective, multidrug-resistant (MDR) (resistant to at least rifampin and isoniazid) TB and extensively drug-resistant (XDR) (resistant to at least rifampin, isoniazid, fluoroquinolones and a second-line injectable agent) TB have had far lower treatment success rates at $56 \%$ and $39 \%$, respectively (1). To improve MDR/XDR-TB treatment success and shorten the duration of treatment, new regimens comprised of doseoptimized new and repurposed anti-TB drugs are needed.

The Nix-TB trial recently showed that a novel, short-course oral regimen comprised of bedaquiline (B), pretomanid ( $\mathrm{Pa}$ ) and linezolid (L) given for 26 weeks produced favorable longterm outcomes in 98 (90\%) of 109 patients with MDR/XDR-TB who had no other treatment options (2). Despite these promising efficacy results, the majority of participants in the trial had to temporarily discontinue and/or reduce the dose of linezolid, due to toxicities including myelosuppression, peripheral neuropathy, or optic neuropathy $(2,3)$. Strategies to mitigate these linezolid-induced toxicities without compromising the efficacy of BPaL would enhance the field implementation of the potentially transformative BPaL regimen by reducing the intensity of toxicity monitoring and the need for adjustments to regimen composition. However, despite recent guidelines ranking linezolid as a preferred agent for treatment of MDR/XDR-TB, there is not consensus on the optimal dosing strategy to balance efficacy and toxicity.

The narrow therapeutic window of linezolid and the long treatment durations required for TB therapy make it difficult to identify a single dose or dosing schedule that optimizes efficacy while minimizing toxicity (2-5). Both its activity against Mycobacterium tuberculosis (M. 
tuberculosis) and its treatment-limiting toxicities appear "time-dependent". Hematological, and possibly neuropathic, toxicities are most closely associated with the proportion of the dosing interval for which concentrations exceed the $\mathrm{IC}_{50}$ for mitochondrial protein synthesis ( $\mathrm{T}_{>\mathrm{MPS} 50}$ ) or its surrogate, $C_{\min }(6-12)$. Likewise, the activity of linezolid against actively replicating $M$. tuberculosis, both in an in vitro hollow fiber model of TB and in an acutely infected BALB/c mouse model of TB, is most closely associated with time above MIC (T>MIC) $(6,13)$. However, we recently found that when bacterial growth is suppressed by the mouse adaptive immune response or cotreatment with a clinically relevant dose of pretomanid, the bactericidal effect of linezolid becomes less dependent on $T_{>M I C}(13)$, suggesting that, when combined with pretomanid and other strong companion drugs, the same weekly dose of linezolid may be dosed more intermittently to limit $\mathrm{T}_{>M P S 50}$-driven toxicity without significant loss of efficacy. These findings led us to hypothesize that, in the context of the BPaL regimen, thrice-weekly administration of linezolid would have similar efficacy and less hematological toxicity than the same total weekly dose of linezolid administered daily. We further hypothesized that "front-loaded" regimens with daily linezolid dosing at a dose equivalent to $1200 \mathrm{mg}$ daily in humans for the first 1-2 months prior to switching to thrice-weekly administration of the same dose (i.e., lowering both total weekly dose and dosing frequency) would be the optimal strategy for maximizing efficacy while minimizing toxicity.

We set out to test the above dosing strategy hypotheses in a murine model of TB, first using $\mathrm{C} 3 \mathrm{HeB} / \mathrm{FeJ}$ mice, which are prone to development of necrotizing granulomatous lung lesions that better represent the pathology of active TB in humans (14-21), and then using the 
45 An alternative dosing strategy of administering linezolid for only the first 2 months, then

46

47

48

continuing with BPa only, which is being tested in the ZeNix trial (ClinicalTrials.gov Identifier:

NCT03086486), was also assessed.

\section{RESULTS}

MICs. Linezolid MIC against both strains was $0.5 \mu \mathrm{g} / \mathrm{mL}$ by agar proportion, while median MIC by broth microdilution was $1 \mu \mathrm{g} / \mathrm{mL}$ against H37Rv and $0.5 \mu \mathrm{g} / \mathrm{mL}$ against HN878. Bedaquiline MIC against both strains was $0.03 \mu \mathrm{g} / \mathrm{mL}$ by agar proportion, while median MIC by broth microdilution was $0.25 \mu \mathrm{g} / \mathrm{mL}$ against $\mathrm{H} 37 \mathrm{Rv}$ and $0.125 \mu \mathrm{g} / \mathrm{mL}$ against HN878. Pretomanid MIC by agar proportion was $0.06 \mu \mathrm{g} / \mathrm{mL}$ against $\mathrm{H} 37 \mathrm{Rv}$ and $0.125 \mu \mathrm{g} / \mathrm{mL}$ against HN878, but MICs by broth microdilution were 0.125 and $0.06 \mu \mathrm{g} / \mathrm{mL}$, respectively.

Experiments 1 and 2: Evaluating linezolid dosing strategies in C3HeB/FeJ mice.

Experiment 1: HN878 strain. We set out to test our dosing strategy hypotheses in C3HeB/FeJ mice infected with $M$. tuberculosis HN878, which, in our experience, consistently produces necrotizing granulomatous lung lesions in a larger proportion of mice than $M$. tuberculosis H37Rv. Control mice received BPa alone, while other mice received BPa plus linezolid at $45 \mathrm{mg} / \mathrm{kg}$ or $90 \mathrm{mg} / \mathrm{kg}$ daily (6 days per week [6/7]), to produce plasma AUC values comparable to $600 \mathrm{mg}$ and $1200 \mathrm{mg}$, respectively, in humans (13). To test the hypothesis that more intermittent dosing of linezolid would be equivalent to daily dosing of the same total weekly dose, another group received BPa plus linezolid at $90 \mathrm{mg} / \mathrm{kg}$ thrice weekly (3/7). After 2 months of treatment, unexpectedly, mice receiving BPaL had higher median CFU counts than animals treated with $\mathrm{BPa}$ alone (Figure $1 \mathrm{~A}$ ) suggesting the possibility of antagonism by linezolid when 
67 added to the regimen. None of the CFU count differences between the BPa group and each individual BPaL group was statistically significant. However, when the BPaL arms were combined post hoc to increase the power to detect a difference and compared to BPa alone, BPa alone was significantly more active than BPa plus linezolid $(p=0.026)$. This result indicating antagonism by

71 linezolid was surprising because previous studies in our high-dose aerosol infection model in BALB/c mice have consistently shown that the addition of linezolid increases the efficacy of BPa after 1-3 months of treatment (22). Those studies used $M$. tuberculosis H37Rv as the infecting strain, raising the possibility that this unexpected result could be related to differences in the $M$. tuberculosis strains or to differences in mouse species.

Experiment 2: H37Rv strain. When increasing doses of linezolid were added to BPa in the treatment of $\mathrm{C} 3 \mathrm{HeB} / \mathrm{FeJ}$ mice infected with the H37Rv strain of $M$. tuberculosis in Experiment 2, was significantly more active that BPa (Figure 1B). After these results we moved to the simpler $\mathrm{BALB} / \mathrm{C}$ mouse model to test both the original linezolid dosing strategy hypotheses and to determine whether the contribution of linezolid to the BPaL regimen was dependent on the $M$. tuberculosis strain. infection model in BALB/c mice infected with $M$. tuberculosis H37Rv or HN878. At the start of treatment (D0), mice infected with the H37Rv strain had a mean lung CFU count of $7.8 \log _{10}$ while 
dose of the latter strain. Mice infected with H37Rv received up to 3 months of treatment. BPa was given for the entire treatment duration. Controls received BPa only or BPa plus linezolid at $90 \mathrm{mg} / \mathrm{kg}$ once daily (5 days per week [5/7]) throughout. Test mice received BPa plus linezolid at $45 \mathrm{mg} / \mathrm{kg}$ (5/7) or $90 \mathrm{mg} / \mathrm{kg}$ (3/7), or linezolid was dosed once daily at $45 \mathrm{mg} / \mathrm{kg}$ or $90 \mathrm{mg} / \mathrm{kg}(5 / 7)$ for 1 or 2 months before being stopped or changed to $90 \mathrm{mg} / \mathrm{kg}$ (3/7). Additional controls received the standard drug-susceptible TB regimen of rifampin, isoniazid, pyrazinamide and ethambutol (RHZE).

As expected in mice infected with the H37Rv strain, the addition of daily linezolid to BPa increased the bactericidal activity of the regimen in a dose-dependent fashion (Table 1 and Fig. S1). Furthermore, BPa alone was significantly less bactericidal when compared to any linezolidcontaining arm after 2 and 3 months of treatment (Table 1 and Fig. S2A-S2D). The same total dose of linezolid administered as $45 \mathrm{mg} / \mathrm{kg}(5 / 7)$ or $90 \mathrm{mg} / \mathrm{kg}(3 / 7)$ had similar additive bactericidal effects when combined with $\mathrm{BPa}$, irrespective of whether the intermittent linezolid dosing began at the start of treatment or after 1 month of daily treatment with linezolid at 45 $\mathrm{mg} / \mathrm{kg}$. Likewise, no significant differences in the proportions of mice relapsing were observed when mice received linezolid at $90 \mathrm{mg} / \mathrm{kg}$ (3/7) instead of $45 \mathrm{mg} / \mathrm{kg}$ (5/7), confirming our hypothesis that, when administered with $\mathrm{BPa}$, thrice weekly dosing of linezolid at $1200 \mathrm{mg}$ would not compromise efficacy compared to linezolid $600 \mathrm{mg} \mathrm{5/7} \mathrm{(Table} 1$ and Fig. S2E and S2F) (13). Regimens using linezolid $90 \mathrm{mg} / \mathrm{kg}$ (5/7) (the highest total weekly dose of any regimen), especially for the first 2 months, were the most efficacious (Table 1 and Fig. S2A-S2D). After 2 months of treatment, BPa plus linezolid $90 \mathrm{mg} / \mathrm{kg}(5 / 7)$ treatment was significantly more bactericidal than all other treatment arms except when linezolid $90 \mathrm{mg} / \mathrm{kg}$ (5/7) was discontinued 
111 at 1 month of treatment (Table 1 and Fig. S2A and S2B). After 3 months of treatment, BPa plus

112 linezolid 90 (5/7) was significantly more bactericidal than all linezolid $45 \mathrm{mg} / \mathrm{kg}$ treatment arms

113 except for when linezolid $45 \mathrm{mg} / \mathrm{kg}$ was given for the full duration of treatment (Table 1 and Fig.

114 S2C and S2D).

Interestingly, giving the same total linezolid dose over 2 months by administering linezolid

at $90 \mathrm{mg} / \mathrm{kg} \mathrm{(5/7)} \mathrm{for} \mathrm{just} \mathrm{the} \mathrm{first} \mathrm{month} \mathrm{resulted} \mathrm{in} \mathrm{lower} \mathrm{CFU} \mathrm{counts} \mathrm{at} \mathrm{month} 2$ than using

117 linezolid at $45 \mathrm{mg} / \mathrm{kg}$ (5/7) or $90 \mathrm{mg} / \mathrm{kg}$ (3/7) for 2 months (Table 1 and Fig. S2A-S2D). An

advantage of using linezolid at $90 \mathrm{mg} / \mathrm{kg}(5 / 7)$ was also observed in the proportions of mice

(5/7) for at least the first two months of treatment or linezolid $90 \mathrm{mg} / \mathrm{kg} \mathrm{(5/7)} \mathrm{for} 1 \mathrm{month}$

121 followed by linezolid $90 \mathrm{mg} / \mathrm{kg}$ (3/7) was associated with relapse in only 1-3 out of 15 mice,

122 compared to relapse proportions of 7-11 out of 15 mice when the linezolid dose was $45 \mathrm{mg} / \mathrm{kg}$

123 (5/7) and/or $90 \mathrm{mg} / \mathrm{kg}$ (3/7) (Table 1). The regimen using linezolid $90 \mathrm{mg} / \mathrm{kg}(5 / 7)$ throughout had

124 statistically significantly fewer relapses compared to all groups having 9-11 relapses, even when

125 adjusted for multiple comparisons, while the differences with groups having 7 relapses were only

126 statistically significant before adjustment for multiple comparisons. By pairwise comparisons, the

127 regimens using linezolid $90 \mathrm{mg} / \mathrm{kg} \mathrm{(5/7)} \mathrm{for} \mathrm{just} \mathrm{the} \mathrm{first} 2$ months or for the first month before

switching to $90 \mathrm{mg} / \mathrm{kg}$ (3/7) produced significantly fewer relapses than the regimens using

129 linezolid $45 \mathrm{mg} / \mathrm{kg} \mathrm{(5/7)} \mathrm{or} 90 \mathrm{mg} / \mathrm{kg}$ (3/7) throughout, although the difference was not quite

130 statistically significant $(p=0.06)$ when comparing $1 B$ PaL90qd/BPaL90qod $(3 / 15$ relapses) to BPaL45qd

131 (9/15 relapses). Furthermore, 2 of the 3 mice relapsing after 3 months of the 1BPaL90qd/BPaL90qod

132 regimen had only a single CFU isolated (Fig. S2F). Taken together, these data support the 
hypothesis that initial dosing of linezolid at $90 \mathrm{mg} / \mathrm{kg}$ daily for the first $1-2$ months results in superior efficacy compared to regimens with lower initial linezolid doses.

To assess the impact of linezolid dosing strategy on its hematological toxicity, whole blood was collected for complete blood counts (CBC) after 2 months of treatment in a cohort of uninfected mice treated in parallel with the infected mice. As previously observed (13), daily (5/7) dosing of linezolid at 45 or $90 \mathrm{mg} / \mathrm{kg}$ was associated with decreased red blood cell (RBC) indices (Figure 2A) compared to BPa-treated and/or untreated mice, whereas use of linezolid at 90 $\mathrm{mg} / \mathrm{kg}(3 / 7)$ throughout or after an initial month of linezolid $90 \mathrm{mg} / \mathrm{kg}(5 / 7)$ was not (Figure 3A, $3 \mathrm{~B}$ and $3 \mathrm{C}$ ). These data suggest that decreasing linezolid dosing frequency, even after the first month of daily treatment, is associated with lower hematological toxicity. As in our previous study, platelet counts were not significantly affected (Figure 2D) (13).

To confirm that the antagonism of linezolid on BPa observed in $\mathrm{C} 3 \mathrm{HeB} / \mathrm{FeJ}$ mice infected with the HN878 strain was bacterial strain-dependent, using a different mouse model, we gave BALB/C mice infected with M. tuberculosis HN878 BPa alone or in combination with linezolid given at $45 \mathrm{mg} / \mathrm{kg}$ (5/7), $90 \mathrm{mg} / \mathrm{kg}$ (5/7) or $90 \mathrm{mg} / \mathrm{kg}$ (3/7) for 2 months, or with linezolid at 90 $\mathrm{mg} / \mathrm{kg}$ (5/7) for 1 month before being either stopped or switched to $90 \mathrm{mg} / \mathrm{kg}$ (3/7). Contrary to results from experiments using the $\mathrm{H} 37 \mathrm{Rv}$ strain in $\mathrm{BALB} / \mathrm{c}$ and $\mathrm{C} 3 \mathrm{HeB} / \mathrm{FeJ}$ mice, but consistent with results against the $\mathrm{HN} 878$ strain in $\mathrm{C} 3 \mathrm{HeB} / \mathrm{FeJ}$ mice, the addition of linezolid did not increase the activity of BPa against the HN878 strain (Table 2 and Fig. S3). Rather, linezolid antagonized $\mathrm{BPa}$ in what appeared to be a dose-dependent fashion. After 1 month of treatment, mean CFU counts were significantly higher among mice receiving BPa plus linezolid at $90 \mathrm{mg} / \mathrm{kg}(5 / 7)$ or every other day compared to those receiving BPa alone (Table 2 and Fig. S3A). After 2 months of 
155 treatment, many mice, including all mice receiving BPa alone, were culture-negative, but all

156 groups receiving linezolid had at least one mouse with detectable CFU counts (Table 2 and Fig.

157 S3B). H37Rv-infected mice treated with BPa plus linezolid $90 \mathrm{mg} / \mathrm{kg}$ (5/7) for 2 months

158 experienced a reduction in bacterial burden of approximately $6.5 \log _{10}$ CFU (Table 1), compared

159 to a decrease of $6.2 \log _{10}$ CFU with the same treatment in HN878-infected mice (Table 2). This

160 suggested that, despite the antagonistic effect of linezolid on BPa in HN878-infected mice, the

161 BPaL regimen remained highly effective.

Experiment 4: Evaluating interactions between drug components in the BPaL regimen and the

influence of bacterial strain in BALB/c mice. To better understand the interactions between the

component drugs and their contribution to the BPaL regimen, including the apparent bacterial

(50 mg/kg) or a T>MIC $_{\text {(100 }} \mathrm{mg} / \mathrm{kg}$ ) similar to that observed in patients taking $200 \mathrm{mg}$ daily (23).

174 addition of pretomanid at $100 \mathrm{mg} / \mathrm{kg}$ or either dose of linezolid had no significant effect.

175 However, the addition of either dose of linezolid to pretomanid alone or to BPa resulted in 
177 was $100 \mathrm{mg} / \mathrm{kg}$ was the 3-drug combination more active than bedaquiline alone (Figure 3A and $1783 B)$.

The activity of bedaquiline alone was greater in mice infected with HN878 than those infected with H37Rv, rendering all HN878-infected mice culture-negative after 2 months of treatment (Figure 3C and 3D). As observed with $\mathrm{H} 37 \mathrm{Rv}$, addition of pretomanid at $50 \mathrm{mg} / \mathrm{kg}$ antagonized bedaquiline, while addition of pretomanid at $100 \mathrm{mg} / \mathrm{kg}$ or linezolid at $90 \mathrm{mg} / \mathrm{kg}$ had no significant effect. Samples from mice receiving bedaquiline plus linezolid at $45 \mathrm{mg} / \mathrm{kg}$ were contaminated during plating and could not be assessed. In stark contrast to results with H37Rv infection, no combination of pretomanid and linezolid was additive against HN878 and the addition of either linezolid dose to BPa was clearly antagonistic irrespective of the pretomanid dose. However, despite the differing contributions and interactions of the 3 component drugs, the full 3-drug BPaL regimen performed quite similarly against both $M$. tuberculosis strains. mutants, we enumerated CFU isolated on bedaquiline-containing and pretomanid-containing plates after 2 months of treatment (Fig. S4, S5 and Table S1). Consistent with prior observations

192 (24), bedaquiline resistance (defined as $\geq 1 \%$ of total CFU) was observed in 2 of 5 H37Rv-infected mice treated with bedaquiline alone. Addition of linezolid at $45 \mathrm{mg} / \mathrm{kg}$ or pretomanid at $50-100$ $\mathrm{mg} / \mathrm{kg}$ prevented growth on bedaquiline-containing plates except for a single CFU on bedaquiline-containing plates for 2 mice or 1 mouse, respectively. Addition of both pretomanid 196 and linezolid prevented recovery of any CFU on bedaquiline plates. Interestingly, no CFU were 197 recovered on bedaquiline plates from any mouse infected with HN878 except for one mouse receiving $\mathrm{BPaL}$ with pretomanid $50 \mathrm{mg} / \mathrm{kg}$ and linezolid $90 \mathrm{mg} / \mathrm{kg}$, the most antagonistic 
combination. Also consistent with prior observations, pretomanid resistance was selected in all mice given pretomanid alone (25), although 2 of 5 H37Rv-infected mice given pretomanid 50 $\mathrm{mg} / \mathrm{kg}$ harbored only 0.02 and $0.1 \%$ resistant CFU. Addition of bedaquiline with or without linezolid prevented recovery of CFU on pretomanid plates except for a single mouse receiving bedaquiline and pretomanid at $100 \mathrm{mg} / \mathrm{kg}$ with $2 \mathrm{CFU}$ on pretomanid plates. However, addition of linezolid only to pretomanid suppressed pretomanid resistance more effectively in HN878infected mice (resistance in only 3 of 20 mice receiving any PaL combination) compared to H37Rvinfected mice (resistance in 13 of 20) $(p=0.003)$ despite greater additive effects of the combination against the susceptible bacterial population in H37Rv-infected mice (Fig. S4, S5 and Table S1).

Recently Pang et al. found antagonism between linezolid and bedaquiline in 13 out of 20 Chinese XDR-TB isolates using an in vitro checkerboard assay (26). To determine whether such antagonism might also occur in our HN878 strain, a member of the East Asian lineage, we performed 3 in vitro checkerboard assays to assess the interaction of linezolid and bedaquiline against both the H37Rv and HN878 strains. The first study used turbidity as the readout. Against the $\mathrm{H} 37 \mathrm{Rv}$ strain, fractional inhibitory concentration index $(\mathrm{FICl})$ values ranged from 1.06 to 1.5 . Against the $\mathrm{HN} 878$ strain, the $\mathrm{FICl}$ range was 1 to 1.25 . The next 2 studies used the Alamar blue assay, as in the study by Pang et al (26). Against the H37Rv strain, the $\mathrm{FICl}$ values were 0.56 to 1.25. Against the $\mathrm{HN} 878$ strain, the $\mathrm{FICl}$ values ranged from 0.75 to 2.25 . All of these values fall in the "indifferent" or "no interaction" range (>0.5-4) (27). Therefore we did not identify antagonism between bedaquiline and linezolid against the H37Rv nor the HN878 strain in our in vitro checkerboard analysis. Notably, across these studies, the bedquiline MIC averaged 1 or 2 
221 dilutions lower against the HN878 strain compared to the H37Rv strain, consistent with the

222 greater efficacy of bedaquiline observed against HN878 compared to H37Rv in vivo.

223

224

225

226

227

228

229

230

231

232

233

234

235

236

237

238

239

240

241

\section{DISCUSSION}

The potent sterilizing activity of the $\mathrm{BPaL}$ regimen and the contribution of each component drug was first discovered using a mouse model of TB (28). In the subsequent Nix-TB clinical trial, BPaL administered for 6 months resulted in an unprecedented $90 \%$ treatment success rate in therapeutically destitute patients with MDR/XDR-TB (2). Despite its promising efficacy as a short-course oral therapy for MDR/XDR-TB, use of the BPaL regimen is complicated by the dose- and duration-dependent toxicity of linezolid, which necessitated a linezolid holiday and/or dose reduction in most participants in the trial (2). No consensus exists on the optimal dosing strategy for linezolid to balance its efficacy and toxicity in TB treatment, whether in the context of the BPaL regimen or in other regimens (29). In the Nix-TB trial, linezolid was initiated at a daily dose of $1200 \mathrm{mg}$, but the dose was commonly reduced to $600 \mathrm{mg}$ daily to manage toxicity, and linezolid was ultimately discontinued before the end of treatment in approximately one-third of participants. At least 2 clinical trials are underway to test different linezolid dosing strategies in the BPaL regimen. The TB-PRACTECAL trial (ClinicalTrials.gov Identifier: NCT02589782) is studying linezolid $600 \mathrm{mg}$ daily for 16 weeks, followed by $300 \mathrm{mg}$ daily or 600 mg every other day for the remaining 8 weeks of treatment. The ZeNix trial (ClinicalTrials.gov Identifier: NCT03086486) is studying linezolid at either $1200 \mathrm{mg}$ or $600 \mathrm{mg}$ daily, with both doses administered for either the entire 26 weeks or the first 9 weeks only. 
Previous pre-clinical studies and limited clinical data have suggested alternative dosing strategies for linezolid. Summarizing results from in vitro hollow fiber system models of TB (6, 30,

31) and from our BALB/c mouse models, we recently proposed that, while linezolid efficacy is driven by $T_{>M I C}$ when $M$. tuberculosis is growing exponentially in the absence of constraints, its efficacy is not as dependent on $T_{>M I c}$ when M. tuberculosis multiplication is restricted by the host immune response or by combining with strong companion drugs (13). This led us to hypothesize that, when linezolid is combined with $\mathrm{BPa}$, linezolid could be dosed at $1200 \mathrm{mg}$ thrice weekly rather than $600 \mathrm{mg}$ daily without loss of efficacy, provided that the same or similar total weekly

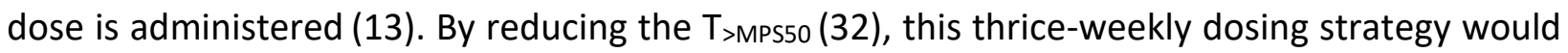

251 be expected to reduce the risk of MPS-driven toxicity. However, because linezolid 1200 mg once

252 daily is likely to be more bactericidal than linezolid $600 \mathrm{mg}$ once daily or $1200 \mathrm{mg}$ thrice weekly

253 at the outset of treatment when M. tuberculosis replication is higher and before bedaquiline and 254 pretomanid have reached steady state $(6,22,32,33)$, we also hypothesized that "front-loading" 255 the regimen with linezolid at $1200 \mathrm{mg}$ once daily before changing to $1200 \mathrm{mg}$ thrice weekly after 256 1-2 months would be the most effective dosing strategy by increasing efficacy without 257 significantly increasing the risk of toxicity compared to thrice-weekly linezolid throughout. A 258 small case series suggesting mitigation of linezolid toxicity upon switching from 800 mg daily to $1200 \mathrm{mg}$ thrice weekly further supports the front-loading concept (34). 
$264 \mathrm{mg} / \mathrm{kg}$ thrice weekly was associated with reduced bactericidal and sterilizing activity, although it

265 did again prevent reductions in RBC indices. Importantly, front loading the linezolid dosing with

$26690 \mathrm{mg} / \mathrm{kg}$ daily before switching to $90 \mathrm{mg} / \mathrm{kg}$ thrice weekly was more effective than linezolid 90

$267 \mathrm{mg} / \mathrm{kg}$ thrice weekly throughout and was as effective as $90 \mathrm{mg} / \mathrm{kg}$ daily throughout, albeit with

268 lesser effects on RBC indices than the last regimen. It is noteworthy that simply stopping linezolid

269 after the first 2 months was as effective as continuing linezolid. This strategy of stopping linezolid

270 at 2 months was initially suggested by a prior study in mice (22) and is currently being evaluated

271 in the ZeNix trial. Our studies therefore provide additional evidence that stopping linezolid at 9

272 weeks in the ZeNix trial will most likely produce results similar to treating with linezolid for the

273 entire treatment duration, while also minimizing toxicity since linezolid-induced toxicities are

274 increasingly frequent after 2 months of treatment. However, continuing linezolid at a safer but

275 still active dose may have advantages, particularly for protecting against the emergence of

276 resistance to bedaquiline and/or pretomanid. Out of the 3 drugs in the BPaL regimen, linezolid

277 has the highest barrier to resistance $(35,36)$. Indeed, prolonged administration of linezolid as

278 functional monotherapy in XDR-TB patients was associated with selection of linezolid-resistant

279 bacteria in only a minority of participants $(3,5)$. This suggests that continuing linezolid after the

280 first 2 months could reduce the risk of emergent resistance to bedaquiline or pretomanid during

281 treatment, an effect that the ZeNix trial may not be sufficiently powered to detect. With

282 increasing evidence of baseline and acquired resistance to bedaquiline or nitroimidazole drugs

283 among MDR/XDR-TB isolates (37-41), including acquired bedaquiline resistance in 1 of 105

284 participants treated with BPaL in a per protocol analysis, it may be prudent to keep linezolid in 
285 the regimen for the entire treatment duration but to change the dosing frequency after 1-2

286

287

288

289

290

291

292

293

294

295

296

297

298

299

300

301

302

303

304

305

306 months to thrice weekly to maintain efficacy but minimize toxicity.

We were surprised to find a decrease in efficacy when linezolid was added to BPa to treat infection with M. tuberculosis strain HN878 as opposed to the increase in efficacy observed when linezolid was added to BPa against H37Rv strain infection. Through our detailed study of the 1-, 2- and 3-drug combinations in the BPaL regimen, we found that the drugs interacted in and contributed to the regimen in different ways. Specifically, both bedaquiline and linezolid were significantly more active as monotherapy against the HN878 strain compared to the H37Rv strain, a finding that may be at least partly explained by the higher MICs in 7H9 media against the latter strain. A recent in vitro checkerboard study from Beijing reported antagonism between bedaquiline and linezolid against the majority of clinical XDR-TB isolates tested (26). However, we did not find such antagonism or evidence that the 2-drug pair of bedaquiline and linezolid interacted differently against H37Rv and HN878 in our in vitro checkerboard assay or in mice. Although pretomanid monotherapy did not exhibit differential effects, the interaction of linezolid with pretomanid was quite different between the two strains in BALB/c mice in that the combination of PaL was more active than pretomanid or linezolid alone against the H37Rv strain but not against the HN878 strain. This interaction of pretomanid and linezolid is what appeared to predict whether addition of linezolid to BPa would increase or decrease the efficacy. These results demonstrating a bacterial strain-dependent contribution of linezolid to the BPaL regimen raise the question of whether the observed differences are attributable to lineage-specific differences such that differential contribution of linezolid or differential activity of the regimen overall could be observed in geographic regions with high prevalence of isolates from the Beijing 
subfamily and/or East Asian lineage to which the HN878 strain belongs. Although it was out of scope for the current report, we plan to investigate whether similar differential contributions of linezolid in the regimen in mice occur with additional clinical isolates from the Euro-American and East Asian lineages. Another consideration is that the less favorable interaction of bedaquiline and/or pretomanid with linezolid is only observed at higher multiples of bactericidal exposures that were only reached against the HN878 strain owing to its greater susceptibility to bedaquiline and linezold. Such a mechanism may not be apparent in in vitro checkerboard studies based on growth inhibition but may be explored further through in vitro time-kill studies. In the end, it is important to note that, despite the strain-dependent differential contribution of linezolid to the regimen, the overall efficacy of the BPaL regimen was quite similar in BALB/C mice, irrespective of the infecting strain, because of the superior efficacy of bedaquiline against the HN878 strain. Moreover, despite not additive activity with pretomanid against the HN878 strain, linezolid was at least as effective at reducing selection of pretomanid-resistant mutants against this strain.

Our study has limitations. The pathological hallmarks of human pulmonary TB are caseating lung lesions and cavities. Although it provided the first evidence of the exceptional sterilizing activity of the $\mathrm{BPaL}$ regimen (22), the BALB/c mouse TB model used here produces only cellular lung lesions, and virtually all infecting bacilli reside intracellularly. We endeavored to test our dosing strategy hypotheses in a C3HeB/FeJ mouse TB model, which exhibits caseating lung lesions that may enable better representation of drug distribution into such lesions and activity against large extracellular bacterial populations in caseum $(18,42)$, but the unanticipated antagonism observed when $L$ was added to BPa against the infecting HN878 isolate confounded 
our results. We successfully tested our dosing strategy hypotheses in the simpler BALB/c mouse model. Our intriguing results showing differential strain-dependent contributions of linezolid to the BPaL regimen are clearly limited by the use of only two strains. Further studies of additional strains from the East-Asian and Euro-American lineages will be necessary to determine whether these differences are lineage-dependent. Lastly, while we hypothesize that there may be clinical benefit in retaining linezolid beyond two months of treatment to prevent resistance, that hypothesis was not tested in these experiments.

\section{METHODS}

Mycobacterial strains. Experiments were conducted using M. tuberculosis H37Rv or HN878, as indicated. Unless otherwise noted, cultures were grown in Middlebrook 7H9 broth supplemented with $10 \%$ oleic acid-albumin-dextrose-catalase (OADC; Difco, Detroit, MI) and $0.05 \%$ Tween 80 (Sigma, St. Louis, MO). MICs were determined head-to-head against each strain using both the agar proportion method on Middlebrook 7H11 agar (performed once) and the microbroth dilution method in Middlebrook 7H9 medium without Tween 80 (Fisher, Pittsburgh, PA) (performed thrice for bedaquiline and linezolid, once for pretomanid).

Aerosol infection model using BALB/c mice. All animal procedures were approved by the Animal Care and Use Committee of Johns Hopkins University. Six-week-old female BALB/c mice were used. Using an inhalation exposure system (Glas-Col, Terre Haute, IN) mice were infected with a log-phase culture of M. tuberculosis H37Rv or HN878 with an optical density at $600 \mathrm{~nm}$ of approximately 1.0 for a target dose of approximately $4 \log _{10}$ CFU implanted in the lungs (22). After aerosol infection, mice were randomized into treatment groups ( 5 mice per treatment arm per time point during treatment and 15 mice per arm for relapse assessment, when evaluated). 
351 Untreated mice were sacrificed (i) the day after infection to quantify CFU implanted in the lungs,

352 (ii) at initiation of treatment to determine pretreatment CFU counts, and (iii) 28 days post-

353 infection to count CFU in untreated controls. Dosing (D0) started 14 days after infection.

354 Aerosol infection model using C3HeB/FeJ mice.

355 HN878 strain. Eight-week-old female C3HeB/FeJ mice were infected in a similar manner to

356 BALB/c mice, except that lower infectious doses were used. A 1:400 dilution of an M. tuberculosis

357 HN878 culture grown for 5 days in 7 H9 media without Tween 80 was passed through a 25 gauge

358 needle 3 times prior to aerosol infection. The infection was repeated 4 weeks later using the

359 same protocol. After infection, body weight was monitored as an indication of disease

360 progression. One cohort of mice with accelerated weight loss (defined as body weight $\leq 25 \mathrm{~g}$ )

361 started treatment 1 week after the second infection, after being randomized (4 mice per arm)

362 into treatment arms. The remaining mice were held for an additional 4 weeks before being

363 randomized into treatment arms (18-26 mice/ arm) and initiated on treatment. Untreated mice

364 were sacrificed (i) the day after each infection to quantify CFU implanted in the lungs, and (ii) at

365 initiation of treatment to determine pretreatment CFU counts.

366 H37Rv strain. Frozen and titrated M. tuberculosis H37Rv stock was diluted at 1:30 by 7H9 media

367 with Tween 80 and used for infection, with the goal of implanting approximately $2.5 \log _{10}$ CFU.

368 Treatment started 4 weeks after infection. Untreated mice were sacrificed same as in the HN878

369 study.

370 Chemotherapy. Rifampin, isoniazid, pyrazinamide and ethambutol were obtained from Sigma

371 and formulated in distilled water. Pretomanid, linezolid, bedaquiline were provided by the Global

372 Alliance for TB Drug Development. Pretomanid was prepared in the CM-2 (cyclodextrin micelle) 
373 formulation, linezolid in $0.5 \%$ methylcellulose, and bedaquiline in an acidified $20 \%$

374 hydroxypropyl- $\beta$-cyclodextrin solution, as previously described $(28,43)$. Dosing formulations

375 were prepared weekly and stored at $4^{\circ} \mathrm{C}$. All drugs except linezolid were administered once daily

3765 days per week (5/7). In Experiment 1, linezolid was administered 3 or 6 days per week (3/7 or

377 6/7). In Experiments 2 and 4, linezolid was administered 5 days per week (5/7). In Experiment 3,

378 linezolid was administered 3 or 5 days per week (3/7 or 5/7). All drugs were given by gavage.

379 Pretomanid and bedaquiline were given at least 4 hours before linezolid (44). RHZE was given

380 once daily with rifampin administered 1 hour prior to HZE. Doses used were as follows:

381 bedaquiline $25 \mathrm{mg} / \mathrm{kg}$, pretomanid 50 or $100 \mathrm{mg} / \mathrm{kg}$ (as indicated), rifampin $10 \mathrm{mg} / \mathrm{kg}$, isoniazid

$38210 \mathrm{mg} / \mathrm{kg}$, pyrazinamide $150 \mathrm{mg} / \mathrm{kg}$ and ethambutol $100 \mathrm{mg} / \mathrm{kg}$. Linezolid doses were 45 or 90

$383 \mathrm{mg} / \mathrm{kg}$, or, in Experiment 2, were 25, 50 or $100 \mathrm{mg} / \mathrm{kg}$ (all as indicated).

384 Assessment of treatment efficacy. Treatment efficacy was assessed using lung CFU counts at 1,

3852 and/or 3 months of treatment and, in Experiment 3, the proportion of mice with culture-

386 positive relapse, defined as $\geq 1$ CFU detected after plating the entire lung homogenate obtained

3873 months after treatment completion. CFU counts were determined by plating neat samples and

388 serial 10 -fold dilutions of lung homogenates on OADC-enriched selective 7H11 agar (Difco)

389 supplemented with $0.4 \%$ activated charcoal as previously described $(45,46)$ to reduce carryover

390 effects. Plates were incubated for at least 6 weeks before final CFU counts were obtained. In

391 Experiment 4, the selection of drug-resistant bacteria after 2 months of treatment was

392 determined by plating aliquots of the lung homogenates in parallel on 7H11 agar with or without

393 bedaquiline $0.06 \mu \mathrm{g} / \mathrm{mL}$ or pretomanid $2 \mu \mathrm{g} / \mathrm{mL}$ to determine the proportion of the total CFU

394 recovered that was resistant to either drug. 
Assessment of treatment toxicity. Blood was collected by cardiac puncture from a separate cohort of uninfected mice treated for 2 months alongside infected mice in Experiment 4. Complete blood counts $(C B C)$ were performed in the Johns Hopkins Phenotyping Core, as previously described (13).

Statistical analysis. Mouse lung CFU counts were log transformed before analysis. In Experiment 1 median CFU counts for BPa and BPaL groups were compared using a nonparametric t-test with were compared using nonparametric analysis with Kruskal-Wallis post-test. In Experiments 3 and

Fisher's Exact test. Group means for CBC parameters were compared using the same test.

In vitro checkerboard assay. M. tuberculosis HN878 and H37Rv were grown in complete 7H9 media with Tween 80 then diluted with $7 \mathrm{H} 9$ media without Tween 80 until a turbidity of $\mathrm{OD}_{600}=$ 1.0 was reached. $100 \mathrm{uL}$ of bacterial suspension was added to each well of 96-well polystyrene plates. Serial 2-fold dilutions were made for both drugs, starting at $4 \mu \mathrm{g} / \mathrm{mL}$ for linezolid and 0.96

$411 \mu \mathrm{g} / \mathrm{mL}$ for bedaquiline. $50 \mu \mathrm{L}$ each drug concentration was added per well to reach a final volume

412 of $200 \mu \mathrm{L}$. Three studies were performed, each of which was 14 days in duration. The first study 413 readout was turbidity. The next two studies used the Alamar blue assay. After 13 days of 414 incubation, $25 \mu \mathrm{L}$ of $0.02 \%$ resazurin solution was added per well and the color was recorded 24 415 hours later. The MIC was defined as the lowest concentration at which a color change from blue 
to pink was not seen. Assessment of drug interactions was based on calculation of the $\mathrm{FICl}$ values

417 using the following equation:

$\mathrm{FICl}=(\mathrm{MIC}$ of $\mathrm{L}$ in combination / MIC of $\mathrm{L}$ alone $)+(\mathrm{MIC}$ of $\mathrm{B}$ in combination / MIC of $\mathrm{B}$ alone $)$

Results for each interaction were defined as synergistic if $\mathrm{FICl}$ values were $\leq 0.5$, indifferent if $\mathrm{FICl}$

value was between 0.5 and 4, antagonistic if $\mathrm{FICl}$ value was $>4$ (27).

\section{ACKNOWLEDGMENTS}

This work was supported by the National Institutes of Health (R01-Al-111992, U19-Al-142735)

authors would also like to thank Si-Yang Li for his help with the mouse model experiments.

\section{REFERENCES}

428

429

430

431

432

433

434

435

436

437

438

439

440

441

442

443

444

445

446

1. WHO. 2018. Global tuberculosis report 2018. /entity/tb/publications/global_report/en/index.html.

2. Conradie F, Diacon AH, Ngubane N, Howell P, Everitt D, Crook AM, Mendel CM, Egizi E, Moreira J, Timm J, McHugh TD, Wills GH, Bateson A, Hunt R, Van Niekerk C, Li M, Olugbosi M, Spigelman M. 2020. Treatment of Highly Drug-Resistant Pulmonary Tuberculosis. N Engl J Med 382:893-902.

3. Lee M, Lee J, Carroll MW, Choi H, Min S, Song T, Via LE, Goldfeder LC, Kang E, Jin B, Park H, Kwak H, Kim H, Jeon HS, Jeong I, Joh JS, Chen RY, Olivier KN, Shaw PA, Follmann D, Song SD, Lee JK, Lee D, Kim CT, Dartois V, Park SK, Cho SN, Barry CE, 3rd. 2012. Linezolid for treatment of chronic extensively drug-resistant tuberculosis. N Engl J Med 367:15081518.

4. Wasserman S, Meintjes G, Maartens G. 2016. Linezolid in the treatment of drugresistant tuberculosis: the challenge of its narrow therapeutic index. Expert Rev Anti Infect Ther 14:901-915.

5. Lee M, Song T, Kim Y, Jeong I, Cho SN, Barry CE. 2015. Linezolid for XDR-TB - Final Study Outcomes. New England Journal of Medicine 373:290-291.

6. Brown AN, Drusano GL, Adams JR, Rodriquez JL, Jambunathan K, Baluya DL, Brown DL, Kwara A, Mirsalis JC, Hafner R, Louie A. 2015. Preclinical Evaluations To Identify Optimal Linezolid Regimens for Tuberculosis Therapy. MBio 6:e01741-01715. 
447 7. Cattaneo D, Orlando G, Cozzi V, Cordier L, Baldelli S, Merli S, Fucile S, Gulisano C, Rizzardini G, Clementi E. 2013. Linezolid plasma concentrations and occurrence of drugrelated haematological toxicity in patients with gram-positive infections. Int J Antimicrob Agents 41:586-589.

8. Pea F, Viale P, Cojutti P, Del Pin B, Zamparini E, Furlanut M. 2012. Therapeutic drug monitoring may improve safety outcomes of long-term treatment with linezolid in adult patients. J Antimicrob Chemother 67:2034-2042.

9. Song $T$, Lee M, Jeon HS, Park Y, Dodd LE, Dartois V, Follman D, Wang J, Cai Y, Goldfeder LC, Olivier KN, Xie Y, Via LE, Cho SN, Barry CE, 3rd, Chen RY. 2015. Linezolid Trough Concentrations Correlate with Mitochondrial Toxicity-Related Adverse Events in the Treatment of Chronic Extensively Drug-Resistant Tuberculosis. EBioMedicine 2:16271633.

10. Matsumoto K, Shigemi A, Takeshita A, Watanabe E, Yokoyama Y, Ikawa K, Morikawa N, Takeda Y. 2014. Analysis of thrombocytopenic effects and population pharmacokinetics of linezolid: a dosage strategy according to the trough concentration target and renal function in adult patients. Int J Antimicrob Agents 44:242-247.

11. Flanagan S, McKee EE, Das D, Tulkens PM, Hosako H, Fiedler-Kelly J, Passarell J, Radovsky A, Prokocimer P. 2015. Nonclinical and pharmacokinetic assessments to evaluate the potential of tedizolid and linezolid to affect mitochondrial function. Antimicrob Agents Chemother 59:178-185.

12. McKee EE, Ferguson M, Bentley AT, Marks TA. 2006. Inhibition of Mammalian Mitochondrial Protein Synthesis by Oxazolidinones. Antimicrob Agents Chemother 50:2042-2049.

13. Bigelow KM, Deitchman AN, Li SY, Barnes-Boyle K, Tyagi S, Soni H, Dooley KE, Savic R, Nuermberger EL. 2020. Pharmacodynamic Correlates of Linezolid Activity and Toxicity in Murine Models of Tuberculosis. J Infect Dis10.1093/infdis/jiaa016:jiaa016.

14. Dartois V. 2014. The path of anti-tuberculosis drugs: from blood to lesions to mycobacterial cells. Nat Rev Microbiol 12:159-167.

15. Cooper AM. 2015. Mouse Model of Tuberculosis. Cold Spring Harb Perspect Med 5:a018556.

16. Driver ER, Ryan GJ, Hoff DR, Irwin SM, Basaraba RJ, Kramnik I, Lenaerts AJ. 2012. Evaluation of a mouse model of necrotic granuloma formation using $\mathrm{C} 3 \mathrm{HeB} / \mathrm{FeJ}$ mice for testing of drugs against Mycobacterium tuberculosis. Antimicrob Agents Chemother 56:3181-3195.

17. Harper J, Skerry C, Davis SL, Tasneen R, Weir M, Kramnik I, Bishai WR, Pomper MG, Nuermberger EL, Jain SK. 2012. Mouse Model of Necrotic Tuberculosis Granulomas Develops Hypoxic Lesions. J Infect Dis 205:595-602.

18. Lanoix JP, Lenaerts AJ, Nuermberger EL. 2015. Heterogeneous disease progression and treatment response in a $\mathrm{C} 3 \mathrm{HeB} / \mathrm{FeJ}$ mouse model of tuberculosis. Dis Model Mech 8:603-610.

19. Nuermberger E. 2008. Using animal models to develop new treatments for tuberculosis. Semin Respir Crit Care Med 29:542-551.

20. Nuermberger JX, Si-Yang L, Deepak VA, Rokeya T, Kala B-B, Paul JC, Anna MU, Khisimuzi $M$, Nader F, Eric L. 2019. Contribution of Pretomanid to Novel Regimens Containing 
Bedaquiline with either Linezolid or Moxifloxacin and Pyrazinamide in Murine Models of Tuberculosis. Antimicrob Agents Chemother 63:e00021-00019.

21. Ordonez AA, Tasneen R, Pokkali S, Xu Z, Converse PJ, Klunk MH, Mollura DJ, Nuermberger EL, Jain SK. 2016. Mouse model of pulmonary cavitary tuberculosis and expression of matrix metalloproteinase-9. Dis Model Mech 9:779-788.

22. Tasneen R, Betoudji F, Tyagi S, Li SY, Williams K, Converse PJ, Dartois V, Yang T, Mendel CM, Mdluli KE, Nuermberger EL. 2016. Contribution of Oxazolidinones to the Efficacy of Novel Regimens Containing Bedaquiline and Pretomanid in a Mouse Model of Tuberculosis. Antimicrob Agents Chemother 60:270-277.

23. Ahmad Z, Peloquin CA, Singh RP, Derendorf H, Tyagi S, Ginsberg A, Grosset JH, Nuermberger EL. 2011. PA-824 Exhibits Time-Dependent Activity in a Murine Model of Tuberculosis. Antimicrob Agents Chemother 55:239-245.

24. Almeida D, Loerger T, Tyagi S, Li S-Y, Mdluli K, Andries K, Grosset J, Sacchettini J, Nuermberger E. 2016. Mutations in pepQ Confer Low-Level Resistance to Bedaquiline and Clofazimine in Mycobacterium tuberculosis. Antimicrobial Agents and Chemotherapy 60:4590-4599.

25. Rifat D, Li S-Y, loerger T, Lanoix J-P, Lee J, Bashiri G, Sacchettini J, Nuermberger E. 2018. Mutations in Rv2983 as a novel determinant of resistance to nitroimidazole drugs in <em>Mycobacterium tuberculosis</em>. bioRxiv10.1101/457754:457754.

26. Pang Y, Jing W, Lu J, Zong Z, Huo F, Dong L, Dai G, Li Y, Huang H, Chu N. 2019. No in vitro synergistic effect of bedaquiline combined with fluoroquinolones, linezolid, and clofazimine against extensively drug-resistant tuberculosis. Diagn Microbiol Infect Dis 94:361-364.

27. Odds FC. 2003. Synergy, antagonism, and what the chequerboard puts between them. J Antimicrob Chemother 52:1.

28. Tasneen R, Li SY, Peloquin CA, Taylor D, Williams KN, Andries K, Mdluli KE, Nuermberger EL. 2011. Sterilizing activity of novel TMC207- and PA-824-containing regimens in a murine model of tuberculosis. Antimicrob Agents Chemother 55:5485-5492.

29. WHO. 2018. Guidelines for treatment of drug-susceptible tuberculosis and patient care (2017 update). /entity/tb/publications/2017/dstb_guidance_2017/en/index.html.

30. Deshpande D, Srivastava S, Pasipanodya JG, Bush SJ, Nuermberger E, Swaminathan S, Gumbo T. 2016. Linezolid for Infants and Toddlers With Disseminated Tuberculosis: First Steps. Clin Infect Dis 63:S80-87.

31. Srivastava S, Magombedze G, Koeuth T, Sherman C, Pasipanodya JG, Raj P, Wakeland E, Deshpande D, Gumbo T. 2017. Linezolid Dose That Maximizes Sterilizing Effect While Minimizing Toxicity and Resistance Emergence for Tuberculosis. Antimicrob Agents Chemother 61:e00751-00717.

32. Diacon AH, De Jager VR, Dawson R, Narunsky K, Vanker N, Burger DA, Everitt D, Pappas F, Nedelman J, Mendel CM. 2020. Fourteen-Day Bactericidal Activity, Safety, and Pharmacokinetics of Linezolid in Adults with Drug-Sensitive Pulmonary Tuberculosis. Antimicrob Agents Chemother 64:e02012-02019.

33. Nuermberger E. 2016. Evolving strategies for dose optimization of linezolid for treatment of tuberculosis. Int J Tuberc Lung Dis 20:48-51. 
34. Chang K-C, Yew W-W, Cheung S-W, Leung C-C, Tam C-M, Chau C-H, Wen PK-H, Chan RCY. 2013. Can intermittent dosing optimize prolonged linezolid treatment of difficult multidrug-resistant tuberculosis? Antimicrobial agents and chemotherapy 57:34453449.

35. Ismail N, Omar S, Peters R. 2018. Collated data of mutation frequencies and associated genetic variants of bedaquiline, clofazimine and linezolid resistance in Mycobacterium tuberculosis. Data Brief 20:1975-1983.

36. Manjunatha UH, Boshoff H, Dowd CS, Zhang L, Albert TJ, Norton JE, Daniels L, Dick T, Pang SS, Barry CE. 2006. Identification of a nitroimidazo-oxazine-specific protein involved in PA-824 resistance in Mycobacterium tuberculosis. Proc Natl Acad Sci U S A 103:431-436.

37. Fujiwara M, Kawasaki M, Hariguchi N, Liu Y, Matsumoto M. 2018. Mechanisms of resistance to delamanid, a drug for Mycobacterium tuberculosis. Tuberculosis (Edinb) 108:186-194.

38. Xu J, Wang B, Hu M, Huo F, Guo S, Jing W, Nuermberger E, Lu Y. 2017. Primary Clofazimine and Bedaquiline Resistance among Isolates from Patients with MultidrugResistant Tuberculosis. Antimicrob Agents Chemother 61:e00239-00217.

39. Ismail NA, Omar SV, Joseph L, Govender N, Blows L, Ismail F, Koornhof H, Dreyer AW, Kaniga K, Ndjeka N. 2018. Defining Bedaquiline Susceptibility, Resistance, CrossResistance and Associated Genetic Determinants: A Retrospective Cohort Study. EBioMedicine 28:136-142.

40. Villellas C, Coeck N, Meehan CJ, Lounis N, de Jong B, Rigouts L, Andries K. 2017. Unexpected high prevalence of resistance-associated Rv0678 variants in MDR-TB patients without documented prior use of clofazimine or bedaquiline. J Antimicrob Chemother 72:684-690.

41. Andries K, Villellas C, Coeck N, Thys K, Gevers T, Vranckx L, Lounis N, de Jong BC, Koul A. 2014. Acquired resistance of Mycobacterium tuberculosis to bedaquiline. PLoS One 9:e102135.

42. Irwin SM, Driver E, Lyon E, Schrupp C, Ryan G, Gonzalez-Juarrero M, Basaraba RJ, Nuermberger EL, Lenaerts AJ. 2015. Presence of multiple lesion types with vastly different microenvironments in $\mathrm{C} 3 \mathrm{HeB} / \mathrm{FeJ}$ mice following aerosol infection with Mycobacterium tuberculosis. Dis Model Mech 8:591-602.

43. Tyagi S, Nuermberger E, Yoshimatsu T, Williams K, Rosenthal I, Lounis N, Bishai W, Grosset J. 2005. Bactericidal Activity of the Nitroimidazopyran PA-824 in a Murine Model of Tuberculosis. Antimicrob Agents Chemother 49:2289-2293.

44. Williams KN, Brickner SJ, Stover CK, Zhu T, Ogden A, Tasneen R, Tyagi S, Grosset JH, Nuermberger EL. 2009. Addition of PNU-100480 to first-line drugs shortens the time needed to cure murine tuberculosis. Am J Respir Crit Care Med 180:371-376.

45. Ahmad Z, Nuermberger EL, Tasneen R, Pinn ML, Williams KN, Peloquin CA, Grosset JH, Karakousis PC. 2010. Comparison of the 'Denver regimen' against acute tuberculosis in the mouse and guinea pig. J Antimicrob Chemother 65:729-734.

46. Almeida D, Nuermberger E, Tasneen R, Rosenthal I, Tyagi S, Williams K, Peloquin C, Grosset J. 2009. Paradoxical effect of isoniazid on the activity of rifampin-pyrazinamide 
combination in a mouse model of tuberculosis. Antimicrob Agents Chemother 53:41784184.

Table 1. Lung CFU counts assessed during treatment and proportions of mice relapsing after treatment of BALB/c mice infected with M. tuberculosis H37Rv.

\begin{tabular}{|c|c|c|c|c|c|c|}
\hline & \multicolumn{5}{|c|}{ Mean lung $\log _{10}$ CFU count ${ }^{a, c-h}( \pm S D)$} & \multirow{2}{*}{$\begin{array}{l}\text { Proportion of mice } \\
\text { relapsing after } \\
\text { treatment for } \\
3 \text { months }\end{array}$} \\
\hline Regimen $^{b}$ & D-14 & DO & M1 & $\mathrm{M} 2$ & M3 & \\
\hline Untreated & $3.90 \pm 0.12$ & $7.79 \pm 0.24$ & & & & \\
\hline RHZE & & & $5.33 \pm 0.16^{f}$ & $3.59 \pm 0.22^{h}$ & $1.63 \pm 0.14^{h}$ & \\
\hline $\mathrm{BPa}$ & & & $5.46 \pm 0.33^{g}$ & $4.03 \pm 0.32^{h}$ & $2.28 \pm 0.20^{h}$ & $15 / 15^{h}$ \\
\hline $\begin{array}{l}1 \mathrm{BPaL}_{45 \mathrm{qd}} \\
/ \mathrm{BPa}\end{array}$ & & & $4.91 \pm 0.20^{c}$ & $2.43 \pm 0.44^{e, g}$ & $0.90 \pm 0.32^{e, g}$ & \\
\hline $\begin{array}{l}1 \mathrm{BPaL}_{45 \mathrm{qd}} \\
/ \mathrm{BPaL}_{90 \mathrm{qod}}\end{array}$ & & & & $2.70 \pm 0.41^{e, h}$ & $0.86 \pm 0.27^{e, g}$ & $7 / 15^{c}$ \\
\hline $\begin{array}{l}2 \mathrm{BPaL}_{45 \mathrm{qd}} \\
/ \mathrm{BPa}\end{array}$ & & & & $3.04 \pm 0.33^{d, h}$ & $0.98 \pm 0.43^{e, g}$ & $7 / 15^{c}$ \\
\hline $\mathrm{BPaL}_{45 \mathrm{qd}}$ & & & & & $0.59 \pm 0.71^{e}$ & $9 / 15^{f}$ \\
\hline $\mathrm{BPaL}_{90 \text { qod }}$ & & & $5.14 \pm 0.28$ & $2.65 \pm 0.44^{e, h}$ & $0.54 \pm 0.47^{e}$ & $11 / 15^{g}$ \\
\hline $\begin{array}{l}1 \mathrm{BPaL}_{90 \mathrm{qd}} \\
/ \mathrm{BPa}\end{array}$ & & & $4.73 \pm 0.42^{d}$ & $1.84 \pm 0.33^{e}$ & $0.41 \pm 0.28^{e}$ & \\
\hline $\begin{array}{l}1 \mathrm{BPaL}_{90 \mathrm{qd}} \\
/ \mathrm{BPaL}_{90 \mathrm{qod}}\end{array}$ & & & & $2.60 \pm 0.49^{e, h}$ & $0.25 \pm 0.35^{e}$ & $3 / 15^{e}$ \\
\hline $\begin{array}{l}2 \mathrm{BPaL}_{90 q d} \\
/ \mathrm{BPa}\end{array}$ & & & & $1.24 \pm 0.87^{e}$ & $0.26 \pm 0.39^{e}$ & $2 / 15^{e}$ \\
\hline $\mathrm{BPaL}_{90 \mathrm{qd}}$ & & & & & $0.09 \pm 0.19^{e}$ & $1 / 15^{e}$ \\
\hline
\end{tabular}

${ }^{a}$ Time points are shown as days (D-14 or D0) or months (M1, M2 or M3).

$582{ }^{b}$ For regimens with two phases, the phases are separated by a slash and the number at the beginning indicates the

583 duration of the initial phase in months.

$584 \quad$ Indicates a significant difference compared to BPa with a p-value $<0.05$.

$585{ }^{d}$ Indicates a significant difference compared to BPa with a p-value $<0.01$.

586 eIndicates a significant difference compared to BPa with a p-value $<0.001$. 
$587 f$ Indicates a significant difference compared to BPaL90qd with a $\mathrm{p}$-value $<0.05$.

588 gIndicates a significant difference compared to BPaLgoqd with a p-value $<0.01$.

$589{ }^{h}$ Indicates a significant difference compared to BPaLgoqd with a p-value $<0.001$.

590 Table 2. Lung CFU counts assessed during treatment and proportions of mice relapsing after 591 treatment of BALB/c mice infected with M. tuberculosis HN878.

\begin{tabular}{|c|c|c|c|c|}
\hline \multirow[b]{2}{*}{ Regimen } & \multicolumn{4}{|c|}{ Mean lung $\log _{10}$ CFU count ${ }^{a}( \pm S D)$} \\
\hline & D-14 & DO & M1 & $\mathrm{M} 2$ \\
\hline Untreated & $2.95 \pm 0.05$ & $6.48 \pm 0.06$ & & \\
\hline $\mathrm{BPa}$ & & & $2.33 \pm 0.23^{g}$ & $0 \pm 0$ \\
\hline $\mathrm{BPaL}_{45 \mathrm{qd}}$ & & & $2.52 \pm 0.12^{f}$ & $0.24 \pm 0.54$ \\
\hline $\mathrm{BPaL}_{90 \mathrm{qod}}$ & & & $2.64 \pm 0.09^{c, e}$ & $0.31 \pm 0.43$ \\
\hline $1 \mathrm{BPaL}_{90 \mathrm{qd}} / \mathrm{BPa}$ & & & $2.95 \pm 0.21^{d}$ & $0.52 \pm 0.49$ \\
\hline $1 \mathrm{BPaL}_{90 \mathrm{qd}} / \mathrm{BPaL}_{90 \mathrm{qod}}$ & & & & $0.31 \pm 0.43$ \\
\hline $\mathrm{BPaL}_{90 \mathrm{qd}}$ & & & & $0.33 \pm 0.66$ \\
\hline
\end{tabular}

${ }^{a}$ Time points are shown as days (D-14 or D0) or months (M1 or M2).

$593{ }^{b}$ For regimens with two phases, the phases are separated by a slash and the number at the beginning indicates the

594 duration of the initial phase in months.

595 Indicates a significant difference compared to BPa with a $\mathrm{p}$-value $<0.05$.

$596 d$ Indicates a significant difference compared to BPa with a p-value $<0.001$.

597 endicates a significant difference compared to BPaL90qd with a p-value $<0.05$.

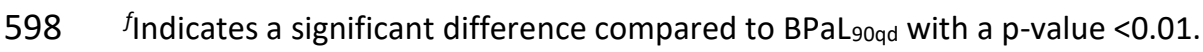

599 Indicates a significant difference compared to BPaL90qd with a p-value $<0.001$. 
A)

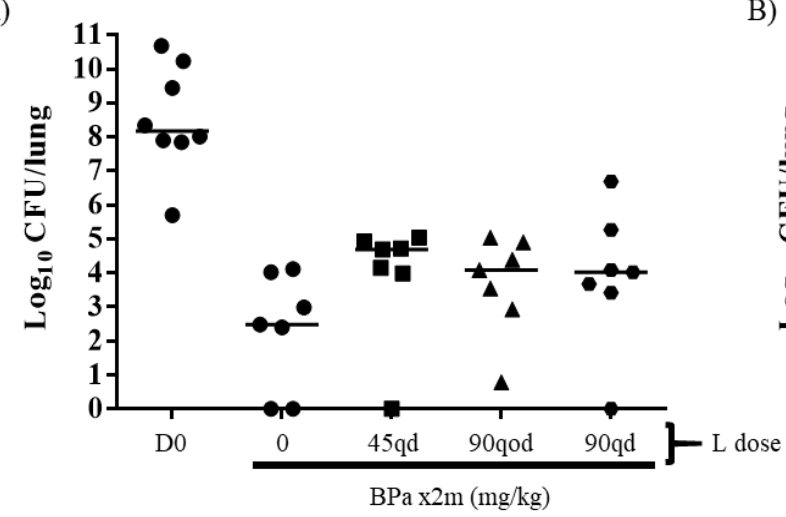

B)

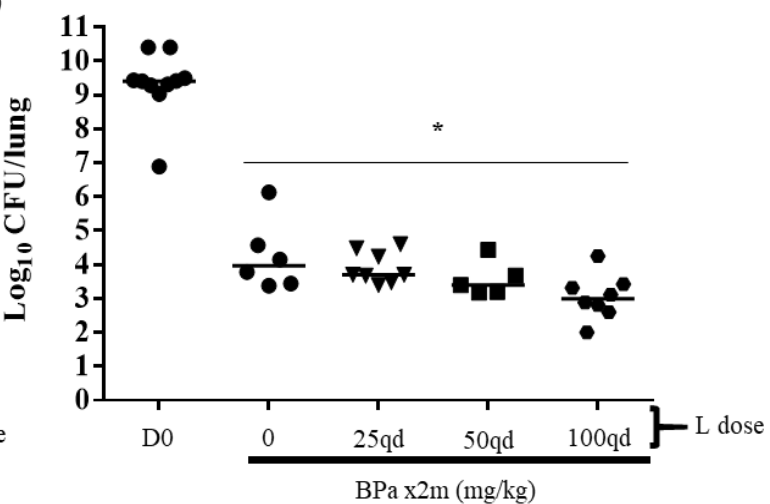

604

605

606

607

608

609

610

611

612

613

614

615

616

617

618

619

620

621

622

623

624

625

626

627

628

629

630
Figure 1. Lung CFU counts in $\mathrm{C} 3 \mathrm{HeB} / \mathrm{FeJ}$ mice after treatment with $\mathrm{BPa}$ with or without $\mathrm{L}$ at differing doses and dosing frequencies. A) Comparison of BPa with or without at $\mathrm{L}$ at $45 \mathrm{mg} / \mathrm{kg}$ qd, L $90 \mathrm{mg} / \mathrm{kg}$ qod, or $\mathrm{L} 90 \mathrm{mg} / \mathrm{kg}$ qd after 2 months of treatment in HN878-infected mice in Experiment 1. B) Comparison of BPa with or without at $L$ at $25 \mathrm{mg} / \mathrm{kg} \mathrm{qd}, 50 \mathrm{mg} / \mathrm{kg}$ qd, or 100 $\mathrm{mg} / \mathrm{kg}$ qd after 1 month of treatment in H37Rv-infected mice in Experiment 2. Abbreviations: $\mathrm{B}$, bedaquiline; Pa, pretomanid; L, linezolid; CFU, colony-forming unit; m, month; qd, once-daily dosing (5/7). Doses used: B $25 \mathrm{mg} / \mathrm{kg} \mathrm{qd,} \mathrm{Pa} 50 \mathrm{mg} / \mathrm{kg}$ (A) or $100 \mathrm{mg} / \mathrm{kg}$ qd (B). *, p-value<0.05. Dark horizontal bars indicate the median value for each group. 

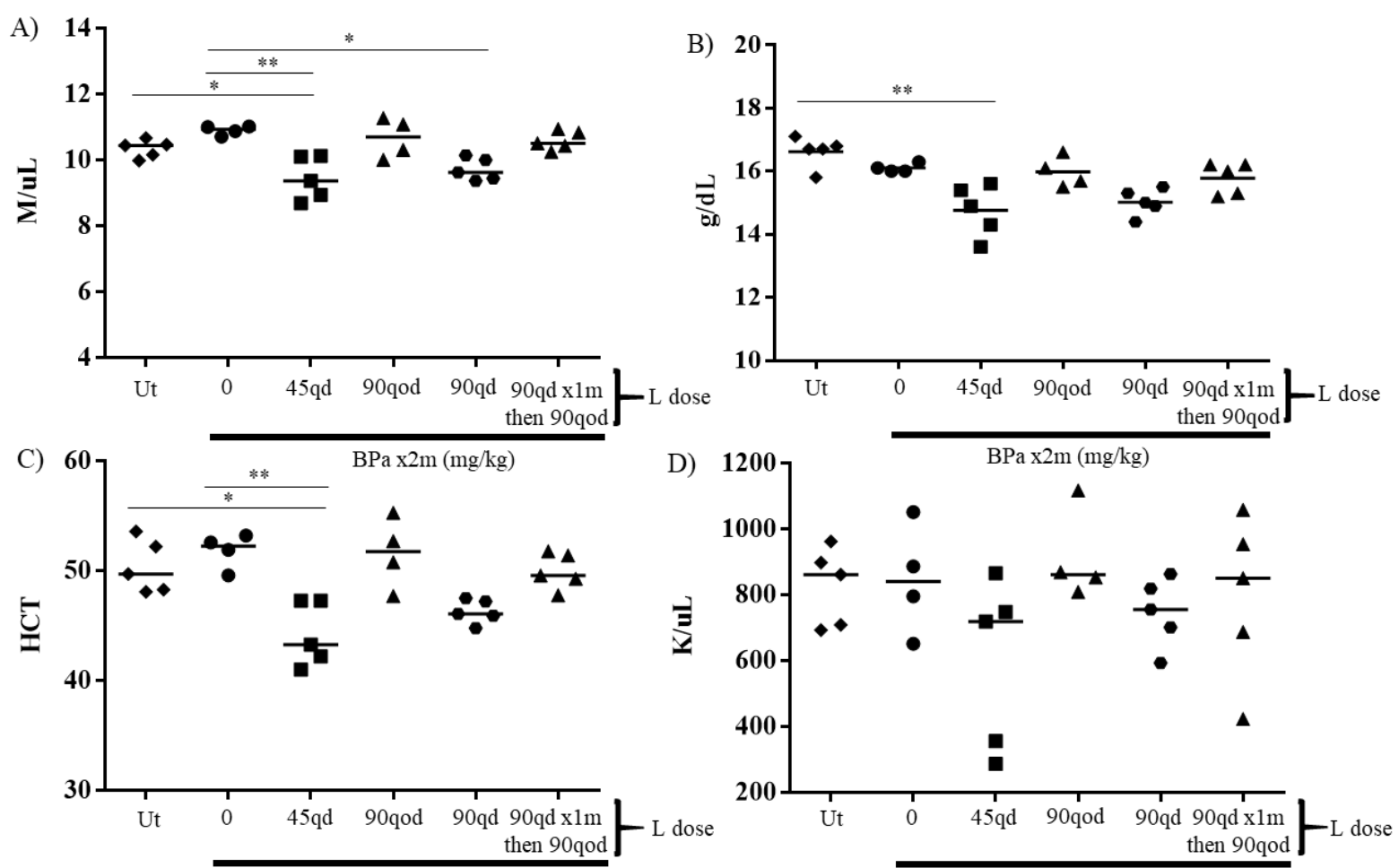

$\mathrm{BPa} \times 2 \mathrm{~m}(\mathrm{mg} / \mathrm{kg})$

$\mathrm{BPa} \times 2 \mathrm{~m}(\mathrm{mg} / \mathrm{kg})$ value $<0.05 ; * *, p$-value $<0.01 ; * * *, p$-value $<0.0001$
637

638

639

640

641

642

643

644

645

646

647

648

649

650

Figure 2. Results of complete blood counts performed in BALB/c mice after 2 months of treatment with $\mathrm{BPa}$ with or without $\mathrm{L}$ at differing dosing frequencies and doses in Experiment 3. A) RBC counts. B) Hemoglobin concentrations. C) Hematocrits. D) Platelet counts. Abbreviations: B, bedaquiline; Pa, pretomanid; L, linezolid; Ut, untreated; m, month; qd, once-daily dosing (5/7); qod, every other day dosing (3/7). Doses used: B $25 \mathrm{mg} / \mathrm{kg}$ qd, Pa $50 \mathrm{mg} / \mathrm{kg}$, L as indicated. *, p- 

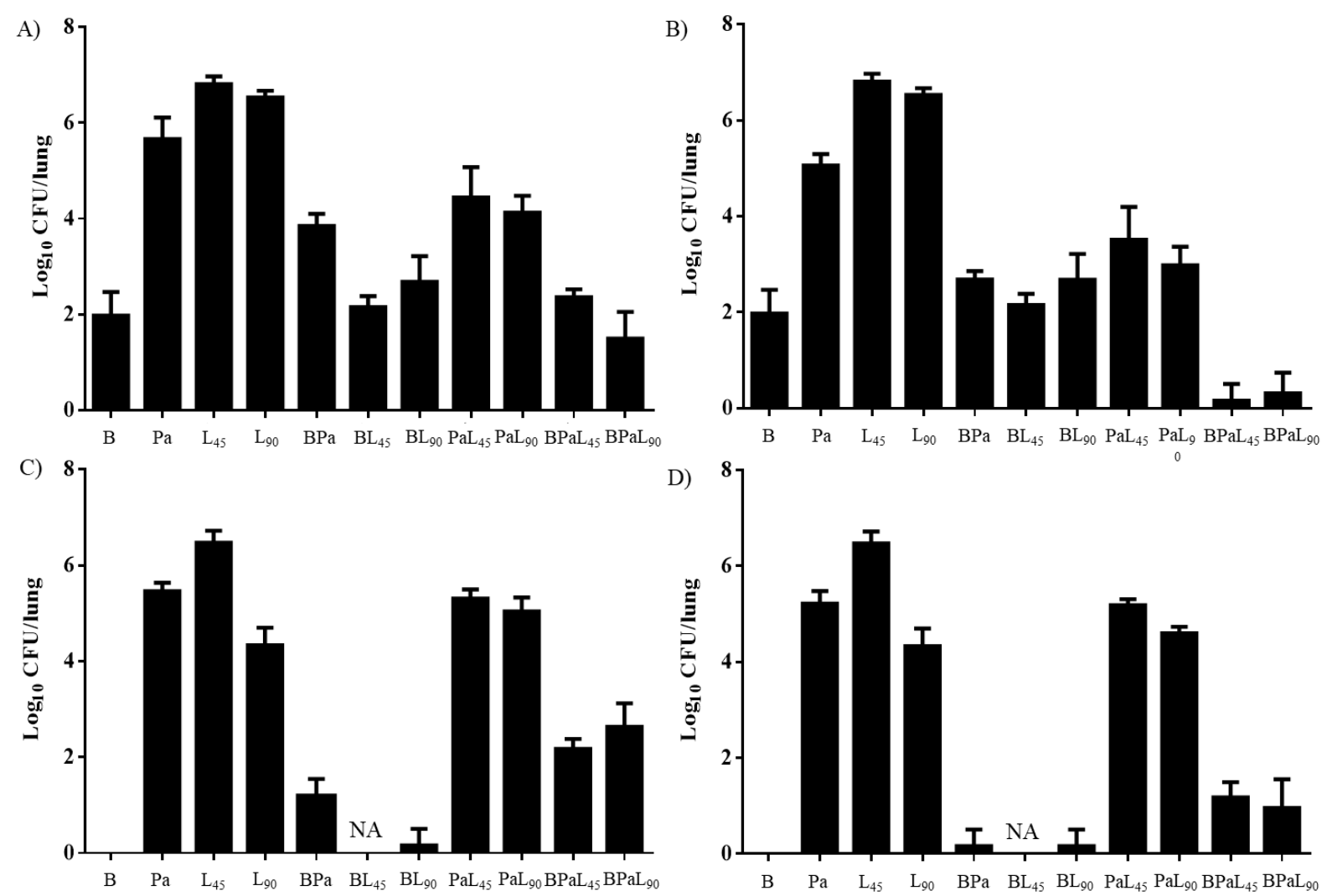

Figure 3. Lung CFU counts after 2 months of treatment with one, two and three drug tuberculosis H37Rv. B) Results for combinations using Pa $100 \mathrm{mg} / \mathrm{kg}$ qd in mice infected with $M$. tuberculosis H37Rv. C) Results for combinations using Pa $50 \mathrm{mg} / \mathrm{kg}$ qd in mice infected with $M$. tuberculosis HN878. D) Results for combinations using Pa $100 \mathrm{mg} / \mathrm{kg}$ qd in mice infected with $M$.

658 forming unit; qd, once-daily dosing (5/7), NA, not available due to contamination of samples. 